\title{
Nintedanib in Severe Idiopathic Pulmonary Fibrosis: Effectiveness through Compassion
}

\author{
Argyrios Tzouvelekis $^{\mathrm{a}, \mathrm{b}} \quad$ Demosthenes Bouros ${ }^{\mathrm{a}}$ \\ a First Academic Department of Pneumonology, Hospital for Diseases of the Chest, "Sotiria", Medical School, \\ National and Kapodistrian University of Athens, Athens, Greece; ${ }^{b}$ Division of Immunology, Biomedical Sciences \\ Research Center "Alexander Fleming", Athens, Greece
}

Idiopathic pulmonary fibrosis (IPF) is the most common idiopathic form of pulmonary fibrosis that affects approximately 5 million people worldwide, leading to death in more than 100,000 patients each year, the same as breast cancer [1]. In 2014, the worldwide approval of 2 antifibrotic agents, nintedanib and pirfenidone, for the treatment of IPF has finally shifted the therapeutic dial. Nintedanib is a multikinase inhibitor and phosphatase activator that has been shown to exert a therapeutic impact on disease progression and acute exacerbations in 3 randomized controlled trials (RCTs) enrolling approximately 1,500 patients with IPF [2-4]. Nintedanib also displayed an acceptable safety profile with common side effects, including diarrhea, leading to drug discontinuation in $20 \%$ of the cases [2-4]. Phase III RCTs set the basis for drug approval and are considered to be at the decisive level of evidence-based medicine. On the other hand, results from RCTs are not generalizable considering that they have been characterized by high screening failure rates $(65 \%$ in the pirfenidone and $29 \%$ in the nintedanib trials) due to stringent enrolment criteria. This has led to the exclusion of a substantial minority of patients commonly encountered in every day clinical practice, namely those with severe lung function impairment.

\section{KARGER}

(c) 2018 S. Karger AG, Basel

E-Mail karger@karger.com

www.karger.com/res
For this reason and to avoid providing patients with a drug of limited clinical reality, it has been suggested to complement RCTs with pragmatic trials that enroll patients who are more representative of the everyday clinical practice and treated under "routine" conditions. To address these concerns, many pharmaceutical companies implemented open-label extension studies to ensure continued access of a study drug to patients who have benefited during the trial until the drug receives approval and becomes commercially available. Nevertheless, these approaches are still limited by selection biases, since they only include patients initially enrolled in the parent phase III trials, leaving outside patients suffering from terminal diseases simply because they do not fulfill enrolment criteria. Compassionate use programs offer a pivotal solution in these situations, since they allow prelaunch drug access to patients with terminal diseases in an inexpensive, ethical, and compliant fashion.

In this issue of Respiration, Harari et al. [5] provide us with the first prospective real-world clinical study on the effectiveness of nintedanib in patients with IPF and severe functional impairment based on a compassionate use program. The authors enrolled 41 patients with an FVC\%predicted $\leq 50 \%$ and a DLCO $\%$ predicted $\leq 35 \%$ 
from multiple centers across Italy and demonstrated that nintedanib exerted therapeutic benefits after 6 months of treatment compared to the pretreatment period, as indicated by an almost 9\% improvement in DLCO\%predicted. Further analysis revealed no significant differences in other functional parameters before and after nintedanib treatment.

The study exhibits a number of major attributes that add knowledge to the existing literature. This is the first prospective data of nintedanib effectiveness into the realworld clinical practice that highlights the necessity of expanding its therapeutic spectrum to a substantial minority of patients with severe disease. The study complements data from the open-label large extension trial INPULSIS-ON that demonstrates similar therapeutic effects of nintedanib in patients with IPF, irrespective of disease severity [6]. It also validates other real-world observational studies based on compassionate use programs including a recent one from our study group, which demonstrated similar therapeutic profiles in patients with IPF of all severity groups [7-11].

On the other hand, current data presents with some limitations that should be interpreted cautiously. A substantial minority $(40 \%)$ of the patients enrolled in the study failed to complete DLCO maneuver due to disease severity and was therefore excluded from analysis. In relation to this, nintedanib did not exert any beneficial effects on FVC\% predicted. Considering that all patients enrolled in the study had available FVC data, one could speculate that therapeutic effects of nintedanib on DLCO could have been diluted, if missing data had been imputed and not censored. Moreover, the study was underpowered and severely hampered by a relatively short followup period; yet, it should be underlined that even in the INPULSIS-ON interim analysis published in 2016 the number of patients with severe IPF under nintedanib treatment was also limited $(n=24)$ [6]. Finally, the authors did not include patients who failed to complete $>6$ months of treatment due to drug toxicity, indicating a potential selection bias.

Despite the above limitations, the study by Harari et al. [5] stresses the idea that treatment guidelines for lifethreatening diseases with dismal prognosis, such as IPF, should not be solely based on studies with the stricter methodology design but they should also take into consideration the consistency of a drug's safety and efficacy in the context of less restrictive but more generalizable study types. More importantly, it highlights the importance of compassionate use or early-access programs to bridge the fundamental gap from the time a drug has been approved to the time it is commercially available to patients, especially for disease paradigms, such as IPF, that have a relatively short survival and limited as well as expensive treatment options. In a world of skepticism, compassionate use initiatives offer hope to patients and strengthen their bonds with physicians, while at the same time they subject drugs into the ultimate "crash test" and expand their efficacy to real-world effectiveness $[12,13]$.

\section{References}

1 Raghu G, Collard HR, Egan JJ, Martinez FJ, Behr J, Brown KK, et al: An official ATS/ERS/ JRS/ALAT statement: idiopathic pulmonary fibrosis: evidence-based guidelines for diagnosis and management. Am J Respir Crit Care Med 2011;183:788-824.

-2 Richeldi L, du Bois RM, Raghu G, Azuma A, Brown KK, Costabel U, et al: Efficacy and safety of nintedanib in idiopathic pulmonary fibrosis. N Engl J Med 2014;370:2071-2082.

-3 Richeldi L, Costabel U, Selman M, Kim DS, Hansell DM, Nicholson AG, et al: Efficacy of a tyrosine kinase inhibitor in idiopathic pulmonary fibrosis. N Engl J Med 2011;365: 1079-1087.

-4 Tzouvelekis A, Yu G, Lino Cardenas CL, Herazo-Maya JD, Wang R, Woolard T, et al: SH2 domain-containing phosphatase-2 is a novel antifibrotic regulator in pulmonary fibrosis. Am J Respir Crit Care Med 2017;195:500514.
5 Harari S, Caminati A, Poletti V, Confalonieri M, Gasparini S, Lacedonia D, et al; for the ILDINET (Interstitial Lung Diseases Italian Network): A real-life multicenter national study on nintedanib in severe idiopathic pulmonary fibrosis. Respiration 2018, DOI: 10.1159/000487711.

6 Wuyts WA, Kolb M, Stowasser S, Stansen W, Huggins JT, Raghu G: First data on efficacy and safety of nintedanib in patients with idiopathic pulmonary fibrosis and forced vital capacity of $\leq 50 \%$ of predicted value. Lung 2016 ; 194:739-743.

7 Tzouvelekis A, Karampitsakos T, Kontou M, Granitsas A, Malliou I, Anagnostopoulos A, et al: Safety and efficacy of nintedanib in idiopathic pulmonary fibrosis: a real-life observational study. Pulm Pharmacol Ther 2018;49:61-66.

$\checkmark 8$ Bonella F, Kreuter M, Hagmeyer L, Neurohr C, Keller C, Kohlhaeufl MJ, et al: Insights from the German compassionate use program of nintedanib for the treatment of idiopathic pulmonary fibrosis. Respiration 2016;92:98-106.
9 King CS, Nathan SD: POINT: Should all patients with idiopathic pulmonary fibrosis, even those with more than moderate impairment, be treated with nintedanib or pirfenidone? Yes. Chest 2016;150:273-275.

10 Toellner H, Hughes G, Beswick W, Crooks MG, Donaldson C, Forrest I, et al: Early clinical experiences with nintedanib in three UK tertiary interstitial lung disease centres. Clin Transl Med 2017;6:41.

11 Hughes G, Toellner H, Morris H, Leonard C, Chaudhuri N: Real world experiences: pirfenidone and nintedanib are effective and well tolerated treatments for idiopathic pulmonary fibrosis. J Clin Med 2016;5.

12 Bouros D: Current therapy of idiopathic pulmonary fibrosis: primum non noccere! Pneumon 2012;25:259-261.

13 Bouros D: Pirfenidone for idiopathic pulmonary fibrosis. Lancet 2011;377:1727-1729. 